\title{
Ab-initio calculation of optical absorption in semiconductors: A density-matrix description
}

\author{
Ulrich Hohenester ${ }^{\Re}$ \\ Institut für Theoretische Physik, Karl-Franzenz-Universität Graz, Universitätsplatz 5, 8010 Graz, Austria
}

(Dated: May 31, 2001)

\begin{abstract}
We show how to describe Coulomb renormalization effects and dielectric screening in semiconductors and semiconductor nanostructures within a first-principles density-matrix description. Those dynamic variables and approximation schemes which are required for a proper description of dielectric screening are identified. It is shown that within the random-phase approximation the direct Coulomb interactions become screened, with static screening being a good approximation, whereas the electron-hole exchange interactions remain unscreened. Differences and similarities of our results with those obtained from a corresponding GW approximation and Bethe-Salpeter equation Green's function analysis are discussed.
\end{abstract}

PACS numbers: 71.35.Cc,71.45.Gm,71.15.Mb

\section{INTRODUCTION}

The study of the optical properties of nanoscale semiconductor structures, such as small clusters, nanocrystals or polymer chains, is acquiring increasing importance also in view of optoelectronic applications. In these systems, however, the theoretical description of the optical response faces additional difficulties with respect to extended crystals, because correlation effects are known to be enhanced. Besides the single-particle energies and wave-functions for electrons and holes, also an appropriate description of Coulomb interactions between electrons and holes is required. For this reason semiempirical or approximate methods (e.g., based on the effectivemass approximation) were often the only feasible treatment so far. In many cases of interest, however, such methods are not sufficiently accurate and predictive.

A strong impulse towards ab-initio approaches is recently coming from the success of standard Density Functional Theory (DFT) methods to treat extended threedimensional systems, 1 with unit cells that may now include thousands of atoms. In semiconductors, however, such DFT approaches are known to account only insufficiently for Coulomb correlation effects. The main reason for this shortcoming is that the dynamics of electronhole excitations is not only governed by the DFT singleparticle states but also by polarization effects, which are not fully accounted for in a standard DFT calculation. More specifically, in semiconductor carriers polarize their surrounding; in turn, this induced polarization affects both the propagation of carriers (quasiparticle renormalizations) and the Coulomb attraction between optically excited electrons and holes (dielectric screening).

Within the framework of Green's functions it has been known for a long time how to correct for such polarization screening. For instance, Hedin and coworker (see Refs. 2. 3 for a review) showed how to consistently develop a set of equations accounting for Coulomb correlation effects. In lowest order, this set of equations reduces to the calculation of the screened Coulomb potential W, obtained from the solution of Dyson's equation for $\mathrm{W}$ within the random-phase approximation, and the self-energy $\Sigma$ which is calculated within the so-called GW approximation (GWA).23 While the GWA approach provides a strongly improved description of single electrons and holes, the description of optical absorption requires in addition a proper treatment of the attractive Coulomb interaction between the optically excited electron-hole pair. This electron-hole correlation is conveniently accounted for by solving the Bethe-Salpeter equation, which describes the propagation the two-particle Green's function (for details see Ref. 1 and references therein).

On the other hand, within the last couple of years phenomenological semiconductor optics, i.e., the simplified bandstructurf and dielectric screening-description of semiconductors, successful field of research. Here, the main interest is on the description of the nonlinear optical response of semiconductors and semiconductor nanostructures, and of optically induced coherence effects. A particularly transparent description scheme for such systems is provided by the dynamics-controlled truncation of the density-matrix hierarchy 10 Contrary to the Green's function approach, which describes by means of many-body perturbation theory the dynamic propagation of quasiparticles, within the framework of density matrices one selects an appropriately chosen set of one-time correlation functions. If Coulomb interactions conserve the number of electronhole pairs, it becomes possible to rigorously classify these correlation functions in powers of the electric field of the exciting laser, 6 thus providing a consistent description scheme for the optical properties of semiconductors.

If, instead of adopting from the beginning a simplified semiconductor description, we start from the results of a first-principles calculation, the most interesting question arises: How is it possible to describe dielectric screening and Coulomb correlation effects within a first-principles density-matrix description? To the best of our knowledge, up to now this problem has not been addressed in the literature. Its answer, however, would be very interesting for a number of reasons. First, it would be helpful to establish a link between the fields of semiconductor op- 
tics and of first-principles calculations; more specifically, such synthesis would allow the first-principles calculation of non-linear optical properties of semiconductors. Second, the framework of density matrices requires only some basic knowledge of quantum mechanics and statistical physics - in contrast to the framework of Green's functions, which rests on a large machinery of definitions and auxiliary functions. Thus, one might hope that certain types of approximations become more transparent; furthermore, the respective strengths of the two approaches might be helpful in establishing approximation schemes beyond the presently used approaches.

This paper is devoted to the discussion of a firstprinciples calculation of dielectric screening within the framework of density matrices. It will be shown that non-pair conserving Coulomb couplings, whose neglect is at the heart of the dynamics-controlled truncation approach, have to be retained for a proper first-principles density-matrix description of dielectric screening and quasiparticle renormalizations in optically excited semiconductors. Adopting the usual random-phase approximation and keeping our analysis at the lowest level of approximation, we will propose a method for the ab-initio calculation of optical absorption which closely resembles that of a corresponding Green's function analysis: first, the exciton energies $E_{x}$ and wavefunctions $\Psi^{x}$ are obtained from the solutions of the two-particle electron-hole Schrödinger equation, where the electron and hole interact with each other through the screened direct Coulomb term and the unscreened exchange one; second, the optical transition energies are given by $E_{x}$ whose oscillator strengths are calculated from $\Psi^{x}$. As the main difference with respect to the corresponding Green's function analysis, our prescription suggests to use static screening in the calculation of the direct Coulomb term.

We have organized this paper as follows. In Sec. II we present our model Hamiltonian and introduce the framework of density matrices; a short overview over one of the most widely used truncation schemes in phenomenological semiconductor optics, the dynamics-controlled truncation, will be given. Sec. III is devoted to the analysis of dielectric screening within the framework of density matrices; the pertinent dynamic variables and approximation schemes are identified. To keep our analysis as simple as possible, details of our calculations are postponed to Appendices A and B. Finally, in Sec. IV we compare our results with those obtained within the GWA and Bethe-Salpeter-equation approach and draw some conclusions. FInally, in Sec. V we draw some conclusions and summarize our prescription for the ab-initio calculation of optical absorption in semiconductors.

\section{DENSITY MATRICES}

\section{A. Hamiltonian}

Let us assume that the single-particle energies $\epsilon_{a}$ and wavefunctions $\phi_{a}(\boldsymbol{r})$ of the semiconductor system under investigation are obtained from a DFT calculation. Although we shall not be too specific about the details of the underlying ab-initio approach, we assume that the Hartree and exchange interactions are included without approximations: 7 a discussion of differences with respect to the local-density approximation (LDA) will be given below. Let $H_{o}$ denote that part of the Hamiltonian which includes the kinetic energy and the lattice interactions, and $\bar{H}^{\prime}$ that part of the electron-electron Coulomb interactions $H^{\prime}$ which are included through the Hartree, exchange, and correlation potentials $v_{H}+v_{x}+v_{c}$. Then, $\epsilon_{a}$ and $\phi_{a}(\boldsymbol{r})$ are the DFT eigenstates of $\bar{H}_{o}=H_{o}+\bar{H}^{\prime}$, and $\delta H=H^{\prime}-\bar{H}^{\prime}$ are those Coulomb interactions which are not included in the DFT approach (and which will be treated in this paper by means of perturbation theory). The basic Hamiltonian describing the carrier system is thus of the form:

$$
H=\bar{H}_{o}+H_{\mathrm{op}}+\delta H,
$$

with the single-particle Hamiltonian $\bar{H}_{o}$; the lightsemiconductor coupling $H_{\mathrm{op}}$; and the Coulomb interactions $\delta H$ which are not included in DFT. Note that for simplicity in Eq. (11) we have neglected all types of carrierphonon interactions or other environment couplings. In the spirit of the GWA approach, in this work we consider in the subtractions to $\delta H$ only the Hartree and exchange terms, thus ignoring possible double counting of Coulomb correlations $v_{c}$. Hence, with the Fermionic field operators $\boldsymbol{\psi}(\boldsymbol{r})$, which create an electron at position $\boldsymbol{r}$, we obtain:

$$
\begin{aligned}
\delta H & \cong \frac{1}{2} \int d\left(\boldsymbol{r} \boldsymbol{r}^{\prime}\right) \frac{\boldsymbol{\psi}^{\dagger}(\boldsymbol{r}) \boldsymbol{\psi}^{\dagger}\left(\boldsymbol{r}^{\prime}\right) \boldsymbol{\psi}\left(\boldsymbol{r}^{\prime}\right) \boldsymbol{\psi}(\boldsymbol{r})}{\left|\boldsymbol{r}-\boldsymbol{r}^{\prime}\right|} \\
& -\int d\left(\boldsymbol{r} \boldsymbol{r}^{\prime}\right)\left[v_{H}(\boldsymbol{r}) \delta\left(\boldsymbol{r}-\boldsymbol{r}^{\prime}\right)+v_{x}\left(\boldsymbol{r}, \boldsymbol{r}^{\prime}\right)\right] \boldsymbol{\psi}^{\dagger}\left(\boldsymbol{r}^{\prime}\right) \boldsymbol{\psi}(\boldsymbol{r})
\end{aligned}
$$

In the DFT semiconductor groundstate all states below the fundamental bandgap are occupied (valence-band states) and all states above the bandgap are unoccupied (conduction-band states). Next, we transform to the electron-hole picture and introduce the Fermionic field operators $c^{\dagger}$ and $d^{\dagger}$, where: $c_{1}^{\dagger}$ creates an electron in the conduction-band state $\phi_{1}^{\mathrm{e}}(\boldsymbol{r})$ with energy $\epsilon_{1}^{\mathrm{e}}$ (henceforth we use numbers $1,2, \ldots$ to label single-particle states); $d_{2}^{\dagger}$ creates a hole in the valence-band state $\phi_{2}^{\mathrm{h}}(\boldsymbol{r})$ with energy $\epsilon_{2}^{\mathrm{h}}$. Since the hole describes the properties of a missing electron in the valence bands, we adopt the usual definitions that for a valence band state $v$ the corresponding hole state 2 is related through $\epsilon_{2}^{\mathrm{h}}=-\epsilon_{v}$ and 
$\phi_{2}^{\mathrm{h}}(\boldsymbol{r})=\left[\phi_{v}(\boldsymbol{r})\right]^{*}$ (with a properly chosen zero-point energy). With these operators: the single-particle Hamiltonian is of the form $\bar{H}_{o}=\sum_{1} \epsilon_{1}^{\mathrm{e}} c_{1}^{\dagger} c_{1}+\sum_{2} \epsilon_{2}^{\mathrm{h}} d_{2}^{\dagger} d_{2}$; the light-matter coupling within the dipole and rotatingwave approximations is:

$$
H_{\mathrm{op}}=-\frac{1}{2} \mathcal{E}_{o} \sum_{12}\left(e^{i \omega t} \mathrm{M}_{21}^{\mathrm{he}} d_{2} c_{1}+e^{-i \omega t} \mathrm{M}_{12}^{\mathrm{eh}} c_{1}^{\dagger} d_{2}^{\dagger}\right),
$$

with $\mathcal{E}_{o} \exp \pm i \omega t$ the electric field of the exciting laser light and $\mathrm{M}_{12}^{\text {eh }}$ the optical dipole matrix elements; the Coulomb terms can be split into three parts $\delta H=$ $\delta H^{(0)}+\delta H^{(1)}+\delta H^{(2)}$, 1 where $\delta H^{(0)}$ conserves the number of electron-hole pairs, $\delta H^{(1)}$ changes the number of electron-hole pairs by one, and $\delta H^{(2)}$ by two (see Appendix A for details).

\section{B. Density-matrix hierarchy}

Having established our model in Sec. II A, we next discuss how to treat Coulomb correlation effects due to $\delta H$. Within the framework of density matrices the central quantities are the one-time correlation functions $\langle\mathcal{A}\rangle_{t}=\operatorname{tr}\left(\boldsymbol{\rho}_{t} \mathcal{A}\right)$, with $\boldsymbol{\rho}_{t}$ the statistical operator at time $t, \operatorname{tr}()$ denoting the trace over a complete set of basis functions, and $\mathcal{A}$ an operator consisting of $c, d, c^{\dagger}$, and $d^{\dagger}$; for instance, $\left\langle c^{\dagger} c\right\rangle_{t}\left(\left\langle d^{\dagger} d\right\rangle_{t}\right)$ describes the occupation of electron (hole) single-particle states at time $t$, whereas higher-order correlation functions, such as, e.g., $\left\langle c^{\dagger} d^{\dagger} d c\right\rangle_{t}$, account for correlations among two or more carriers. As the central approximation within any density-matrix description one has to restrict oneself to a limited number of correlation functions. The temporal evolution of the correlation fuptions $\langle A\rangle_{t}$ is then provided by Ehrenfest's theorem $\mathbf{3}$ B

$$
\partial_{t}\langle\mathcal{A}\rangle_{t}=\langle[\mathcal{A}, H]\rangle_{t},
$$

which, together with the restriction to a finite number of correlation functions, provides the basis of the framework of density matrices. Before addressing the problem of dielectric screening in Sec. III, in the following we briefly review one of the most commonly used truncation schemes in phenomenological semiconductor optics: The dynamics-controlled truncation 6 This discussion will help us to establish some of the basic concepts and notations.

\section{Dynamics-controlled truncation}

In describing the optical properties of conventional semiconductors one often relies on effective models. Besides a simplified bandstructure description in terms of $\boldsymbol{k} . \boldsymbol{p}$-thpery or effective-mass approximations one usually: 5 employs the envelope-function approximation; neglects the non-pair conserving terms $\delta H^{(1)}$ and $\delta H^{(2)}$; and screens the Coulomb interactions in $\delta H^{(0)}$ by the static dielectric constant (see Sec. III for a first-principles motivation of such approximation). Hence, the remaining terms $\bar{H}_{o}+\delta H^{(0)}$ conserve the number of electrons and holes, and the only source for the creation or destruction of electron-hole pairs is through the light-matter coupling $H_{\mathrm{op}}$; more specifically, inspection of Eq. (3) reveals that the creation (destruction) of electron-hole pairs is through terms of the form $\mathcal{E}_{o} \mathcal{c}^{\dagger} d^{\dagger}\left(\mathcal{E}_{o} d c\right)$. In the pioneering work of Axt and Stahl 10 (see Ref. 6 for a review) the authors first noted that, since any pair of field operators $c^{\dagger} d^{\dagger}$ or $d c$ comes with an electric field $\mathcal{E}_{o}$, the density matrices can be classified according to their power in the electric field.

Thus, in linear optical response there is only one correlation function

$$
Y_{12}=\left\langle d_{2} c_{1}\right\rangle
$$

which is of lowest order in $\mathcal{E}_{o} . Y$ describes the correlation between an optically excited electron and hole (note that $Y$ is a non-equilibrium quantity which is nonzero only in presence of an exciting laser and which vanishes in thermal equilibrium). Its relation to the total interband polarization $\mathcal{P}(t)$ is given through $\mathcal{P}(t)=\sum_{12} \mathrm{M}_{21}^{\text {he }} Y_{12, t}$, with $\mathrm{M}^{\mathrm{eh}}$ the optical dipole matrix elements. Recalling that the optical absorption is related through Maxmell's equation to the total interband polarization $\mathcal{P}(t), 0$ one immediately notes that the microscopic interband polarizations $Y$ completely determine the linear optical response of semiconductors. Their temporal evolution is given by Eq. (4), and we find after some straightforward algebra:

$$
i \dot{Y}_{12}=\left(\epsilon_{1}^{\mathrm{e}}+\epsilon_{2}^{\mathrm{h}}\right) Y_{12}-\frac{1}{2} \mathcal{E}_{o} e^{-i \omega t} \mathrm{M}_{12}^{\mathrm{eh}}-\left(\mathrm{V}_{1 \overline{1}, 2 \overline{2}}^{\mathrm{ee}, \mathrm{h}}-\mathrm{V}_{12, \overline{2} \overline{1}}^{\mathrm{eh}, \mathrm{he}}\right) Y_{\overline{1} \overline{2}},
$$

where we have implicitly assumed summation over barred indices $\overline{1}$ and $\overline{2}$. On the right-hand side of Eq. (6): the first term accounts for the single-particle states available for adding an electron-hole pair; the second term describes the creation of electron-hole pairs through coupling to the light field; finally, the third term accounts for the electron-hole Coulomb interactions (with $\mathrm{V}^{\mathrm{ee}, \mathrm{hh}}$ the direct and $\mathrm{V}^{\text {eh,he }}$ the exchange term), and is responsible for excitonic renormalizations in the optical spectra (which would be absent in a simple-minded singleparticle description).

Eq. (6) is conveniently solved by finding the polarization eigenmodes. The homogeneous part of Eq. (6) is then transformed to angeigenvalue problem ("excitonic eigenvalue problem"), 56 whose solutions provide the exciton energies $E_{x}$ and wavefunctions $\Psi_{12}^{x}$. In the optical spectra the optical transition energies are then provided by $E_{x}$, and the respective oscillator strengths are given by $\left|\sum_{12} \Psi_{12}^{x} \mathrm{M}_{21}^{\text {he }}\right|^{2}$. 


\section{DIELECTRIC SCREENING}

As it is well known that dielectric screening is of central importance for the quantitative description of the optical properties of semiconductors, within an ab-initio approach it would make little sense to directly employ Eq. (6) with the bare (i.e., unscreened) Coulomb matrix elements V. For instance, in conventional semiconductors, such as Si or GaAs, dielectric screening leads to a reduction of the unscreened V's by a factor of approximately ten. We are thus faced with the central question of this work: How can dielectric screening be described within the framework of density matrices?

Quite generally, dielectric screening is a process where a carrier polarizes its surrounding medium. In turn, a second carrier not only feels the bare Coulomb interaction exerted by the first carrier, but also the field produced by this induced polarization cloud. In an electronhole picture, such polarization effects are described by (virtual) excitations of electron-hole pairs which result in microscopic polarization fields. Thus, a proper firstprinciples description of dielectric screening requires besides the pair-conserving Coulomb terms $\delta H^{(0)}$ also the non-pair conserving terms $\delta H^{(1)}$ and $\delta H^{(2)}$.

Inclusion of such terms, however, spoils the concept of the dynamics-controlled truncation of the density-matrix hierarchy, since electron-hole pairs are no longer solely created (destroyed) by the light field but, in addition, also by Coulomb interactions. Thus, it is no longer possible to rigorously classify correlation functions according to their power in the electric field $\mathcal{E}_{o}$. However, since both the light field and the Coulomb interactions create electrons and holes only pairwise, we can still assume that the classification of correlation functions in numbers of electron-hole pairs provides a meaningful concept. For simplicity, in this paper we restrict ourselves to the case of correlation functions with at most two electron-hole pairs, and we show that already at this level of approximation dielectric screening is included.

\section{A. Dynamic variables}

Besides the interband polarizations $Y_{12}$, we hence need the electron (hole) distribution function $C_{12}=\left\langle c_{2}^{\dagger} c_{1}\right\rangle$ $\left(D_{12} \bar{\sigma}\left\langle d_{2}^{\dagger} d_{1}\right\rangle\right)$ and the two-particle correlation functions: 6

$$
\begin{aligned}
& B_{12,34}=\left\langle d_{4} c_{3} d_{2} c_{1}\right\rangle \\
& N_{12,34}=\left\langle c_{3}^{\dagger} d_{4}^{\dagger} d_{2} c_{1}\right\rangle .
\end{aligned}
$$

In analogy to the physical meaning of $Y$ as a measure of the electron-hole correlation and $C(D)$ as the occupation and intraband coherence of single-particle electron (hole) states, we can approximately interpret $B$ as a measure for the coherence between two electron-hole pairs and $N$ as the occupation of electron-hole pairs.
Next, we derive the equations of motion for the various correlation functions. Before doing so, we employ a further approximation. From the analysis of the nonlinear coherent optical response (i.e., the dynamics-controlled truncation at the level of $\mathcal{E}_{o}^{3}$, which involves $Y, C, D, B$, and $N)^{6}$ it is known that the main effect of the carrier distribution functions $C$ and $D$ is to reduce the optical transition rates because of state filling (i.e., Pauli blocking). As we expect such effects to be of minor importance for the problem of our present concern, in the following we shall neglect $C$ and $D$. Thus, our set of dynamic variables is provided by $Y, B$, and $N$.

\section{B. Equations of motion for $Y, B$, and $N$}

The program pursued in the following is the derivation of the equations of motion for $Y, B$, and $N$. This is done by using Ehrenfest's theorem, Eq. (4), together with the definitions for $Y, B$, and $N$, Eqs. (5, 7, 8) (see also Appendix A). After some straightforward algebra we obtain:11

$$
\begin{aligned}
i \dot{Y}_{12} \cong & \left(\epsilon_{1}^{\mathrm{e}}+\epsilon_{2}^{\mathrm{h}}\right) Y_{12}-\frac{1}{2} \mathcal{E}_{o} e^{-i \omega t} \mathrm{M}_{12}^{\mathrm{eh}} \\
& -\left(\mathrm{V}_{1 \overline{1}, 2 \overline{2}}^{\mathrm{ee}, \mathrm{hh}}-\mathrm{V}_{12, \overline{2} \overline{1}}^{\mathrm{eh}, \mathrm{he}}\right) Y_{\overline{1} \overline{2}} \\
& +\left(\mathrm{V}_{1 \overline{1}, 34}^{\mathrm{ee}, \mathrm{eh}}-\mathrm{V}_{3 \overline{1}, 14}^{\mathrm{ee}, \mathrm{eh}}\right) N_{\overline{1} 2,34}+\mathrm{V}_{1 \overline{1}, 43}^{\mathrm{ee}, \mathrm{he}} B_{\overline{1} 2,34} \\
& -\left(\mathrm{V}_{2 \overline{2}, 34}^{\mathrm{hh}, \mathrm{eh}}-\mathrm{V}_{4 \overline{2}, 32}^{\mathrm{hh}, \mathrm{eh}}\right) N_{1 \overline{2}, 34}-\mathrm{V}_{2 \overline{2}, 43}^{\mathrm{hh}, \mathrm{he}} B_{1 \overline{2}, 34}
\end{aligned}
$$

(here and henceforth we implicitly assume summation over all single-particle indices with exception of those appearing on the left-hand side). Comparison with Eq. (5), which was derived by neglecting non-pair conserving Coulomb couplings, reveals the appearance of an additional coupling to the two-particle correlations $B$ and $N$, which is mediated through the non-pair-conserving Coulomb interactions $\delta H^{(1)}$ and $\delta H^{(2)}$. As will be shown in the following, such terms are needed for a proper description of dielectric screening.

Let us discuss this coupling between $Y$ and $B, N$ in slightly more detail. Suppose that the system under investigation is a bulk semiconductor. Hence, the singleparticle states $1,2, \ldots$ consist of a wavevetor $\boldsymbol{k}$ and a band index $n$. As the light field couples electron and hole states with approximately opposite $\boldsymbol{k}$-values, the optically induced interband polarization is of the form $Y_{\boldsymbol{k}}=\left\langle d_{-\boldsymbol{k}} c_{\boldsymbol{k}}\right\rangle$, where for notational simplicity we have dropped all band indices. The coupling $\mathrm{VB}$ in Eq. (9) is then of the form $\sum_{\boldsymbol{p} \boldsymbol{q}} \mathrm{V}(\boldsymbol{q})\left\langle d_{-\boldsymbol{p}+\boldsymbol{q}} c_{\boldsymbol{p}} d_{-\boldsymbol{k}+\boldsymbol{q}} c_{\boldsymbol{k}}\right\rangle$, with a corresponding expression for $\mathrm{V} N$. Hence, these terms describe how the propagation of $Y$ is modified by the presence of polarization fluctuations in the system; as we will show in the following, such fluctuations are induced by the interband polarization $Y$, thus resulting in a selfinteraction-like process where: the interband polarization $Y$ induces polarization fluctuations through the non-pairconserving Coulomb couplings $\delta H^{(1)}$; these fluctuations 
propagate in time, which is described by the equations of motion for $B$ and $N$ (to be derived below); and finally couple back to $Y$, which is described by the terms $\mathrm{VB}$ and $\mathrm{V} N$ in Eq. (9).

Next, we derive the equations of motion for $B$ and $N$. To keep our analysis as simple as possible, we employ from the beginning a number of well-controlled approximations. First, we assume that before arrival of the exciting laser no two-particle correlations $B$ and $N$ are present (as will be discussed below this is only an approximation). Hence, $Y, B, N$ are induced by the light-semiconductor coupling $H_{\mathrm{op}}$, and in linear response it suffices to keep in the dynamics only terms linear in $Y, B, N$ (i.e., we neglect contributions proportional to, e.g., $Y^{2}$ or $Y B$ ). Second, we neglect the light coupling in the equations of motion for $B$ and $N$ (such terms would describe interference terms between dielectric screening and light coupling). Third, we shall emplo the so-called random-phase approximation (RPA):12 13 For $B_{12,34}$ and $N_{12,34}$ we keep only Coulomb terms which scatter one pair of particles 12 (34) and leave the other pair 34 (12) unaffected. In other words, we assume that polarization fluctuations created through light-coupling and Coulomb processes, respectively, move independently of each other. In a $k$-space representation for the polarization fluctuations $\left\langle d_{-\boldsymbol{p}+\boldsymbol{q}} c_{\boldsymbol{p}} d_{-\boldsymbol{k}+\boldsymbol{q}} c_{\boldsymbol{k}}\right\rangle$ and $\left\langle c_{\boldsymbol{p}}^{\dagger} d_{-\boldsymbol{p}+\boldsymbol{q}}^{\dagger} d_{-\boldsymbol{k}+\boldsymbol{q}} c_{\boldsymbol{k}}\right\rangle$ one readily observes that these RPA terms contain all those contributions which involve Coulomb matrix elements $\mathrm{V}(\boldsymbol{q})$ with a momentum exchange of $\boldsymbol{q}$ which is independent of $\boldsymbol{k}$ and $p .13$

With these approximations we then find:

$$
\begin{aligned}
i \dot{B}_{12,34} \cong & \left(\epsilon_{1}^{\mathrm{e}}+\epsilon_{2}^{\mathrm{h}}+\epsilon_{3}^{\mathrm{e}}+\epsilon_{4}^{\mathrm{h}}\right) B_{12,34} \\
& +\left(\mathrm{V}_{12, \overline{2} \overline{1}}^{\mathrm{eh}, \mathrm{he}}-\mathrm{V}_{1 \overline{1}, 2 \overline{2}}^{\mathrm{ee}, \mathrm{hh}}\right) B_{\overline{1} \overline{2}, 34}+\left(\mathrm{V}_{12, \overline{1} \overline{2}}^{\mathrm{eh}, \mathrm{eh}}-\mathrm{V}_{1 \overline{2}, \overline{1} 2}^{\mathrm{eh}, \mathrm{eh}}\right) N_{34, \overline{1} \overline{2}} \\
& +\left(\mathrm{V}_{34, \overline{4} \overline{3}}^{\mathrm{eh}, \mathrm{he}}-\mathrm{V}_{3 \overline{3}, 4 \overline{4}}^{\mathrm{ee}, \mathrm{hh}}\right) B_{12, \overline{3} \overline{4}}+\left(\mathrm{V}_{34, \overline{3} \overline{4}}^{\mathrm{eh}, \mathrm{eh}}-\mathrm{V}_{3 \overline{4}, \overline{3} 4}^{\mathrm{eh}, \mathrm{eh}}\right) N_{12, \overline{3} \overline{4}} \\
& +\left(\mathrm{V}_{1 \overline{1}, 34}^{\mathrm{ee}, \mathrm{eh}}-\mathrm{V}_{3 \overline{1}, 14}^{\mathrm{ee}, \mathrm{eh}}\right) Y_{\overline{1} 2}-\left(\mathrm{V}_{2 \overline{2}, 34}^{\mathrm{hh}, \mathrm{eh}}-\mathrm{V}_{4 \overline{2}, 32}^{\mathrm{hh}, \mathrm{eh}}\right) Y_{1 \overline{2}} \\
& +\left(\mathrm{V}_{3 \overline{3}, 12}^{\mathrm{ee}, \mathrm{eh}}-\mathrm{V}_{1 \overline{3}, 32}^{\mathrm{ee}, \mathrm{eh}}\right) Y_{\overline{3} 4}-\left(\mathrm{V}_{4 \overline{4}, 12}^{\mathrm{hh}, \mathrm{eh}}-\mathrm{V}_{2 \overline{4}, 14}^{\mathrm{hh}, \mathrm{eh}}\right) Y_{3 \overline{4}}+\left(\mathrm{V}_{12,34}^{\mathrm{eh}, \mathrm{eh}}-\mathrm{V}_{14,32}^{\mathrm{eh}, \mathrm{eh}}\right) \\
i \dot{N}_{12,34} & \cong\left(\epsilon_{1}^{\mathrm{e}}+\epsilon_{2}^{\mathrm{h}}-\epsilon_{3}^{\mathrm{e}}-\epsilon_{4}^{\mathrm{h}}\right) N_{12,34} \\
& +\left(\mathrm{V}_{12, \overline{2} \overline{1}}^{\mathrm{eh}, \mathrm{he}}-\mathrm{V}_{1 \overline{1}, 2 \overline{2}}^{\mathrm{ee}, \mathrm{hh}}\right) N_{\overline{1} \overline{2}, 34}+\left(\mathrm{V}_{12, \overline{1} \overline{2}}^{\mathrm{eh}, \mathrm{h}}-\mathrm{V}_{1 \overline{2}, \overline{1} 2}^{\mathrm{eh}, \mathrm{eh}}\right) B_{\overline{1} \overline{2}, 34}^{*} \\
& -\left(\mathrm{V}_{43, \overline{3} \overline{4}}^{\mathrm{he}, \mathrm{eh}}-\mathrm{V}_{\overline{3} 3, \overline{4} 4}^{\mathrm{ee}, \mathrm{hh}}\right) N_{12, \overline{3} \overline{4}}-\left(\mathrm{V}_{43, \overline{4} \overline{3}}^{\mathrm{he}, \mathrm{he}}-\mathrm{V}_{\overline{4} \overline{3}, 4 \overline{3}}^{\mathrm{he}, \mathrm{he}}\right) B_{12, \overline{3} \overline{4}} .
\end{aligned}
$$

Let us analyze the various contributions to these equations in slightly more detail. On the right-hand side of Eq. (10): the first term corresponds to the free propagation of $B$; the terms in the second and third line, respectively, describe Coulomb renormalization processes in the propagation of $B(\mathrm{~V} B)$ and Coulomb couplings between $N$ and $B(\mathrm{VN})$; the terms in the last two lines are the source terms which describe how polarization fluctuations are created through coupling to the interband polarizations $Y$. In addition, there is a term $\left(\mathrm{V}_{12,34}^{\mathrm{eh}, \mathrm{eh}}-\mathrm{V}_{14,32}^{\mathrm{eh}, \mathrm{eh}}\right)$ which describes the buildup of polarization fluctuations even in absence of light couplings. Quite generally, its appearance is not unexpected since up to now we have assumed that the semiconductor groundstate is obtained by simply filling DFT single-particle states. Acting with $\delta H^{(2)}$ on the DFT vacuum, however, we immediately observe that Coulomb interactions create electron-hole pairs and thus lead to renormalizations of the DFT vacuum. As a first approximation, in this paper we assume that such renormalizations are not of crucial importance for the description of dynamic processes in the propagation of $Y$, and we thus neglect this term.

In the equation of motion for $N$, Eq. (11), we observe in analogy to the dynamics of $B$ single-particle contributions (first line) and Coulomb renormalizations and couplings (second and third line), but no source terms are present. Similarly to the semiconductor Bloch equations, 5 which describe the non-equilibrium optical response at the level of two-point functions (i.e., $C, D$, and $Y$ ) and where the light field drives the electron-hole coherence $Y$ which, in turn, acts as a source term for the electron (hole) occupations $C(D)$, we here have the situation that Coulomb coupling first drives the electronhole pair coherence $B$ which, in turn, acts as source for the electron-hole pair occupation $N$.

Eqs. (9 11) are the central equations of this work. Before showing how to solve this set of equations, we introduce two further approximations. First, we keep in Eqs. (10,11) only self-interaction like processes. The electron-hole coherence $Y_{\overline{1} 2}\left(Y_{1 \overline{2}}\right)$ between the states $\overline{1} 2$ $(1 \overline{2})$ is initially created through the coupling to the light field. In a self-interaction process: this coherence is transferred through Coulomb coupling of the electron (hole) to 12 and to a second electron-hole pair 34 [fourth line in Eq. (10)]; the second electron-hole pair 34 propagates in presence of Coulomb renormalizations [third 
lines in Eqs. (10,11)] and the initial pair 12 remains unscattered; finally, this coherence between electron-hole pairs 12 and 34 affects the propagation of $Y$ [third and fourth line in Eq. (9))]. In addition to these selfinteraction like processes, there exist also scattering-like contributions: here either the optically induced coherence between electron-hole pair 34 is transferred through Coulomb coupling to the pair 12 [fifth line in Eq. (10)] or the coherence between pair 12 is scattered to $\overline{1} \overline{2}$ [second lines in Eqs. (10, 11)]. Such terms, which will be neglected in the following, describe higher-order Coulomb terms (within the framework of Green's functions they would correspond to vertex corrections); a more detailed discussion of such scattering contributions has been given in Ref. 13.

Our second approximation concerns the neglect of electron-hole exchange interactions in the dynamics of $B$ and $N$ : in Eqs. (10, 11) we only keep the first Coulomb terms in parentheses. One readily observes in a $k$ space representation that for the polarization fluctua- tions $\left\langle d_{-\boldsymbol{p}+\boldsymbol{q}} c_{\boldsymbol{p}} d_{-\boldsymbol{k}+\boldsymbol{q}} c_{\boldsymbol{k}}\right\rangle$ and $\left\langle c_{\boldsymbol{p}}^{\dagger} d_{-\boldsymbol{p}+\boldsymbol{q}}^{\dagger} d_{-\boldsymbol{k}+\boldsymbol{q}} c_{\boldsymbol{k}}\right\rangle$ these terms correspond to Coulomb interactions $\mathrm{V}(\boldsymbol{q})$ where the exchanged momentum $\boldsymbol{q}$ is independent of the momenta $\boldsymbol{k}$ and $\boldsymbol{p}$; the remaining terms involve exchanged momenta depending on $\boldsymbol{k}$ and $\boldsymbol{p}$. The more general expressions of Eqs. (10,11) which contain both the direct and exchange contributions have been given for the purpose of our later discussion about the screening of the electron-hole exchange interaction.

Details of our solution scheme for Eqs. (10) and (11) are presented in Appendix B. The key to the solution is the Dyson-like character of the equations of motion for $B$ and $N$. In fact, solving Eqs. (10,11) by iteration we observe that the repeated action of $\mathrm{V}(B+N)$ precisely reproduces Dyson's equation for the screened Coulomb potential $\mathrm{W}=\mathrm{V}\left[1+\mathrm{VP}+(\mathrm{VP})^{2}+\ldots\right]=\mathrm{V}+\mathrm{WPV}$; here $P$ is the usual retarded polarization function within random-phase approximation. Finally, we arrive at (for details see Appendix B):

$$
\begin{aligned}
i \dot{Y}_{12} \cong & \left(\epsilon_{1}^{\mathrm{e}}+\epsilon_{2}^{\mathrm{h}}\right) Y_{12}+\mathcal{D}_{1 \overline{1}, \overline{1} \tilde{1}}^{\mathrm{ee}, \mathrm{e}}\left(\omega_{\overline{1} 2}\right) Y_{\tilde{1} 2}+\mathcal{D}_{2 \overline{2}, \overline{2} \tilde{2}}^{\mathrm{hh}}\left(\omega_{1 \overline{2}}\right) Y_{1 \tilde{2}}-\frac{1}{2} \mathcal{E}_{o} e^{-i \omega t} \mathrm{M}_{12}^{\mathrm{eh}} \\
& -\left(\mathrm{V}_{1 \overline{1}, 2 \overline{2}}^{\mathrm{ee}, \mathrm{hh}}+\mathcal{D}_{1 \overline{1}, 2 \overline{2}}^{\mathrm{ee}, \mathrm{hh}}\left(\omega_{\overline{1} 2}\right)+\mathcal{D}_{2 \overline{2}, 1 \overline{1}}^{\mathrm{hh}, \mathrm{ee}}\left(\omega_{1 \overline{2}}\right)-\mathrm{V}_{12, \overline{2} \overline{1}}^{\mathrm{eh}, \mathrm{h}}\right) Y_{\overline{1} \overline{2}}
\end{aligned}
$$

with

$$
\mathcal{D}\left(\boldsymbol{r}, \boldsymbol{r}^{\prime} ; \omega\right)=\int d\left(\boldsymbol{s s}^{\prime} \bar{\omega}\right) \mathrm{W}(\boldsymbol{r}, \boldsymbol{s} ; \omega) \frac{D_{o}\left(\boldsymbol{s}, \boldsymbol{s}^{\prime} ; \bar{\omega}\right)}{\omega-\bar{\omega}+i 0^{+}} \mathrm{V}\left(\boldsymbol{s}^{\prime}, \boldsymbol{r}^{\prime}\right)
$$

[the relation between the real-space and single-particle representations of $\mathcal{D}$ is in analogy to Eq. (A4)]; $D_{o}$ is related to the imaginary part of the polarization function through $D_{o}\left(\boldsymbol{r}, \boldsymbol{r}^{\prime} ; \omega\right)=-\pi^{-1} \Im P\left(\boldsymbol{r}, \boldsymbol{r}^{\prime} ; \omega>0\right)$ [Eqs. (B5, B8)]. A particularly simple expression of Eq. (12) follows for static screening, i.e., for:

$$
\mathcal{D}\left(\boldsymbol{r}, \boldsymbol{r}^{\prime} ; \omega=0\right)=\frac{1}{2}\left[\mathrm{~W}\left(\boldsymbol{r}, \boldsymbol{r}^{\prime} ; 0\right)-\mathrm{V}\left(\boldsymbol{r}, \boldsymbol{r}^{\prime}\right)\right]
$$

where the expression in the second line of Eq. (12) reduces to $\left[\mathrm{W}_{1 \overline{1}, 2 \overline{2}}^{\mathrm{ee}, \mathrm{hh}}(0)-\mathrm{V}_{12, \overline{2} \overline{1}}^{\mathrm{e}}\right] Y_{\overline{1} \overline{2}}$. Thus, in Eq. (12) the $\mathcal{D}$-terms in the first line describe renormalization effects in the propagation of single electrons and holes, respectively, and the terms in the second line account for the Coulomb coupling between the optically excited electronhole pair.

\section{DISCUSSION}

Equation (12) is our final expression. Before analyzing it in more detail, let us briefly recall the approximations that were adopted in its derivation. First, within the spirit of the dynamics-controlled truncation 6 only dynamic variables accounting at most for two electron-hole pairs were considered. The electron and hole distribution functions $C$ and $D$ were neglected, since from related work it is known that their main influence is the blocking of optical transitions due to state filling. Thus, our set of dynamic variables consists of the interband polarizations $Y$, and the two-particle correlations $B$ and $N$ which account for the coherence between electron-hole pairs and their occupation, respectively. We assumed that before arrival of the exciting laser light $Y, B$, and $N$ vanish. In the equations of motion for $B$ and $N$ : we adopted the random-phase approximation 13 which assumes that polarization fluctuations propagate independently of each other; we neglected the direct coupling of $B$ and $N$ to the light field; only self-interaction-like processes were kept; finally, exchange-type interactions were neglected.

Quite generally, none of these approximations is compulsory, and all of them could be lifted without introducing major conceptual modifications. However, we have kept our analysis as simple as possible in order to emphasize the two primary goals of this work: first, the 
identification of those terms and approximations which are required within a density-matrix formalism in order to describe dielectric screening; and second, to discuss the respective differences between our approach and the combined GWA and Bethe-Salpeter equation approach.

Within our density-matrix description, the central quantities are the optically induced interband polarizations $Y$. In general, their knowledge allows the full calculation of the optical absorption spectra. However, as discussed in Sec. IIC, the temporal evolution of $Y$ is in addition governed by the two-particle correlations $B$ and $N$, which, within a self-interaction like process, are induced through the non-pair conserving Coulomb couplings $\delta H^{(1)}$ and $\delta H^{(2)}$; the details of this coupling, Eqs. (9 11), reflect the essential features of dielectric screening where: an electron (hole) polarizes its surrounding medium (described by the source terms for $B)$; in turn, the hole (electron) not only feels the bare Coulomb term exerted by the the first carrier but also the field induced by the polarization cloud [cf. terms in the second line of Eq. (12)]; in addition, this induced polarization cloud also affects the propagation of the carrier [cf. $\mathcal{D}$-terms in the first line of Eq. (12)]. Thus, the screening of the electron-hole interaction and the quasiparticle renormalizations originate from the same physical process.

It is interesting to compare this finding with the respective GWA and Bethe-Salpeter equation result. Let us first concentrate on the case of static screening, Eq. (14). Inspection of the quasiparticle renormalizations of Eq. (12) with the results of the GWA approach 3 reveals that these renormalizations closely resemble the screened exchange and Coulomb hole self-energy terms (note that this comparison is somewhat complicated because of our use of the electron-hole picture and the missing screening of the electron-hole exchange interactions). If dynamic screening is considered, the inelasticities $\omega_{12}$ of $\mathcal{D}\left(\omega_{12}\right)$ in Eq. (12) are given by the differences between the light frequency $\omega$ and the electron-hole transition energies $\epsilon_{1}^{\mathrm{e}}+\epsilon_{2}^{\mathrm{h}}$. Assuming that the exciton states are composed of electron and hole single-particle states with energies close to the bandgap, one immediately notes that $\omega \approx \epsilon_{1}^{\mathrm{e}}+\epsilon_{2}^{\mathrm{h}}$; thus, static screening of the direct Coulomb interactions is expected to be a good approximation. This result is different with respect to the GWA prescription, where the inelasticities of the quasiparticle renormalizations [first line of Eq. (12)] are the quasiparticle energies themselves, and the electron-hole interaction is screened at the optical frequency $\omega, \omega$ (see also Ref. 14 for a discussion of the compensation between effects due to dynamical screening and higher-order vertex corrections; the consequences of such interplay within our present density-matrix description will be discussed elsewhere).

Next, we discuss the screening of the electron-hole exchange interactions $\mathrm{V}^{\text {eh,he }}$. As we saw in the discussion of the screening of the direct Coulomb terms, dielectric screening originates from a process where: the interband polarizations $Y$ induce the electron-hole pair correlations
$B$ and $N$; these induced polarization fluctuations move independently of each other (random-phase approximation); and finally couple back to the propagation dynamics of $Y$. These self-interaction-like processes result in the terms $\mathcal{D}_{1 \overline{1}, 2 \overline{2}}$, Eq. (13), where the vertices $2 \overline{2}$ and $1 \overline{1}$, respectively, reflect the coupling of $Y$ to the electron-hole pair coherence $B$ and the back action of $B, N$ on $Y$; finally, the propagation dynamics of $B$ and $N$ is hidden in $\mathcal{D}$, which has to be determined from the Dyson-like equation for the screened Coulomb potential W. Let us now return to the full expression of Eqs. (10.11) which, within the approximations adopted, contain all possible source terms. One immediately recognizes that neither these source terms nor the coupling of $B, N$ to $Y$, Eq. (9), can reproduce a screening term of the form $\mathcal{D}^{\text {eh,he }}$. Hence, within our approximation scheme (RPA) the electronhole exchange interactions must remain unscreened. Future work will address possible screening contributions and vertex corrections beyond the random-phase approximation.

Finally, we briefly comment on one of the well-known shortcomings of our density-matrix description. From Eqs. (12) and (B5) one readily observes that the unrenormalized single-particle energies $\epsilon^{\mathrm{e}}$ and $\epsilon^{\mathrm{h}}$ enter into the calculation of the screened Coulomb potential and of the polarization function $P$. This result differs from the corresponding GWA and Bethe-Salpeter equation result and is due to our neglect of higher-order correlation functions (i.e., correlations between three or more electronhole pairs). However, it can be shown 15 that inclusion of certain types of Coulomb interactions at this level of many-particle correlations indeed results in a renormalization of the single-particle energies $\epsilon^{\mathrm{e}}$ and $\epsilon^{\mathrm{h}}$. For the sake of brevity, here we shall not present the details of such analysis.

When defining the Coulomb interactions of our starting Hamiltonian, Eq. (2), we assumed that exchange interactions are fully accounted for within the DFT calculations. Apparently, this assumption no longer holds for DFT calculations based on the celebrated local-density approximation (LDA) 1 . Inspection of Eq. (2) reveals that in this case the substracted exchange contributions would be of the form $v_{x}\left(\boldsymbol{r}, \boldsymbol{r}^{\prime}\right) \cong \delta\left(\boldsymbol{r}-\boldsymbol{r}^{\prime}\right) v_{x}^{\mathrm{LDA}}(\boldsymbol{r})$; as consequence, in $\delta H$ only the Hartree contributions with the filled valence band would be cancelled exactly (with no corresponding cancellation for the exchange terms) and the local exchange potential would give rise to terms proportional to $c^{\dagger} d^{\dagger}$ and $d c$. In the equations of motion for the dynamic variables these local-exchange contributions would give rise to additional couplings between variables containing different numbers of electron-hole pairs. Within the spirit of perturbation theory, such couplings are due to the fact that the semiconductor groundstate is not given by simply filling the DFT-LDA states; rather, the non-local exchange interactions give rise to admixtures of excited DFT-LDA states, which, within the electron-hole picture, correspond to dynamic variables accounting for different numbers of electron-hole pairs. 
However, it is well known that in many cases the singleparticle renormalizations to the DFT-LDA states simply lead to a rigid shift of the calculated bandgap (scissor operator) and that the calculated wavefunctions remain unchanged. In this case, the results of our analysis remain valid and only the electron-hole exchange interactions $\mathrm{V}^{\mathrm{eh}}$,he, which correct for the missing Coulomb exchange interactions because of optical excitation, should be computed within the local-density approximation.

\section{CONCLUSIONS AND SUMMARY}

In conclusion, starting from an ab-initio bandstructure calculation we have shown how to describe dielectric screening within a density-matrix description. We have identified those dynamic variables and approximations which are required at the lowest level of approximation (random-phase approximation). We have discussed that static screening is expected to be a good approximation (if contrast to the corresponding Green's function results 1 which seem to favour screening at the optical frequencies). Hence, our analysis suggests calculation of the optical absorption spectra from the solutions of the "excitonic eigenvalue problem":

$$
\left(\bar{\epsilon}_{1}^{\mathrm{e}}+\bar{\epsilon}_{2}^{\mathrm{h}}\right) \Psi_{12}^{x}-\left(\mathrm{W}_{1 \overline{1}, 2 \overline{2}}^{\mathrm{ee}, \mathrm{hh}}(0)-\mathrm{V}_{12, \overline{2} \overline{1}}^{\mathrm{eh}}\right) \Psi_{\overline{1} \overline{2}}^{x}=E_{x} \Psi_{12}^{x},
$$

with $E_{x}$ and $\Psi^{x}$ the exciton energies and wavefunctions, respectively (with proper summation over barred indices). Here, we have assumed that single-particle renormalizations (scissor operator, GW corrections) lead to a rigid shift of the calculated bandgap, with $\bar{\epsilon}_{1}^{\mathrm{e}, \mathrm{h}}$ the renormalized single-particle energies [see Eq. (12) for the more complete expression]; the W's are the Coulomb interactions screened within RPA, where the dielectric function is computed at zero frequency (static-screening approximation) using the renormalized single-particle energies $\bar{\epsilon}_{1}^{\mathrm{e}, \mathrm{h}}$; finally, $\mathrm{V}^{\mathrm{eh}}$,he are the unscreened electron-hole exchange interactions; within LDA, these terms should be computed within the local approximation. In the optical spectra, the optical transition energies are provided by $E_{x}$, and the respective oscillator strengths are given by $\left|\sum_{12} \Psi_{12}^{x} \mathrm{M}_{21}^{\mathrm{he}}\right|^{2}$.

It is hoped that our approach might serve as an alternative to the hitherto used GWA and Bethe-Salpeterequation approaches. Its advantage lies in its conceptual simplicity: only some basic knowledge about statistical mechanics and Heisenberg's equations of motion are required (as opposed to the more sophisticated framework of Green's functions). Finally, this work might help to extend the variety of techniques developed recently within the field of semiconductor optics for the description of the nonlinear optical response to first-principles studies.

\section{Acknowledgments}

I gratefully acknowledge most helpful discussions with Alice Ruini, Marilia Caldas, and Elisa Molinari.

\section{APPENDIX A}

In this Appendix we discuss the transformation of Eq. (2) to the electron-hole picture. The relation between the field operators $\psi$ and $c, d$ is given by:

$$
\boldsymbol{\psi}^{\dagger}(\boldsymbol{r})=\sum_{1}\left[\phi_{1}^{\mathrm{e}}(\boldsymbol{r})\right]^{*} c_{1}^{\dagger}+\sum_{2} \phi_{2}^{\mathrm{h}}(\boldsymbol{r}) d_{2}
$$

Inserting this expression into Eq. (2), we obtain after some straightforward algebra:

$$
\begin{aligned}
\delta H^{(0)} & =\frac{1}{2}\left(\mathrm{~V}_{\overline{1}, \overline{2} 22}^{\mathrm{ee}, \mathrm{ee}} c_{\overline{1}}^{\dagger} c_{\overline{2}}^{\dagger} c_{1} c_{2}+\mathrm{V}_{\overline{1} 1, \overline{2} 2}^{\mathrm{hh}, \mathrm{hh}} d_{\overline{1}}^{\dagger} d_{\overline{2}}^{\dagger} d_{1} d_{2}\right)-\left(\mathrm{V}_{\overline{1} 1, \overline{2} 2}^{\mathrm{ee}, \mathrm{hh}}-\mathrm{V}_{\overline{1} \overline{2}, 21}^{\mathrm{eh}, \mathrm{he}}\right) c_{\overline{1}}^{\dagger} d_{\overline{2}}^{\dagger} d_{2} c_{1} \\
\delta H^{(1)} & =\mathrm{V}_{12, \overline{3} 3}^{\mathrm{eh}, \mathrm{ee}} c_{1}^{\dagger} d_{2}^{\dagger} c_{\overline{3}}^{\dagger} c_{3}-\mathrm{V}_{12, \overline{3} 3}^{\mathrm{eh},{ }_{3}} c_{1}^{\dagger} d_{2}^{\dagger} d_{\overline{3}}^{\dagger} d_{3}+\mathrm{V}_{\overline{3} 3,21}^{\mathrm{ee}, \mathrm{he}} c_{\overline{3}}^{\dagger} c_{3} d_{2} c_{1}-\mathrm{V}_{\overline{3} 3,21}^{\mathrm{hh}, \mathrm{he}} d_{\overline{3}}^{\dagger} d_{3} d_{2} c_{1} \\
\delta H^{(2)} & =\frac{1}{2}\left(\mathrm{~V}_{12,34}^{\mathrm{eh}, \mathrm{eh}} c_{1}^{\dagger} c_{3}^{\dagger} d_{4}^{\dagger} d_{2}^{\dagger}+\mathrm{V}_{21,43}^{\mathrm{he}, \mathrm{he}} d_{2} d_{4} c_{3} c_{1}\right)
\end{aligned}
$$

(we assume an implicit summation over all single-particle indices); the commutation relations between $c, d$ and the various Coulomb terms are: 


$$
\begin{aligned}
& {\left[c_{1}, \delta H^{(0)}\right]=\mathrm{V}_{1 \overline{1}, \overline{3} 3}^{\mathrm{ee}, \mathrm{ee}} c_{\overline{3}}^{\dagger} c_{3} c_{\overline{1}}-\left(\mathrm{V}_{1 \overline{1}, \overline{3} 3}^{\mathrm{ee}, \mathrm{hh}}-\mathrm{V}_{1 \overline{3}, 3 \overline{1}}^{\mathrm{eh}, \mathrm{he}}\right) d_{\overline{3}}^{\dagger} d_{3} c_{\overline{1}}} \\
& {\left[d_{2}, \delta H^{(0)}\right]=\mathrm{V}_{2 \overline{2}, \overline{3} 3}^{\mathrm{hh}} d_{\overline{3}}^{\dagger} d_{3} d_{\overline{2}}-\left(\mathrm{V}_{2 \overline{2}, \overline{3} 3}^{\mathrm{hh}, \mathrm{ee}}-\mathrm{V}_{2 \overline{3}}^{\mathrm{he}, 3 \overline{2}}\right) c_{\overline{3}}^{\dagger} c_{3} d_{\overline{2}}} \\
& {\left[c_{1}, \delta H^{(1)}\right]=\left(\mathrm{V}_{1 \overline{1}, 34}^{\mathrm{ee}, \mathrm{eh}}-\mathrm{V}_{3 \overline{1}, 14}^{\mathrm{ee}, \mathrm{eh}}\right) c_{3}^{\dagger} d_{4}^{\dagger} c_{\overline{1}}-\mathrm{V}_{1 \overline{2}}^{\mathrm{eh}, \overline{3} \mathrm{~h}} d_{\overline{2}}^{\dagger} d_{\overline{3}}^{\dagger} d_{3}+\mathrm{V}_{1 \overline{1}, 43}^{\mathrm{ee}, \mathrm{he}} d_{4} c_{3} c_{\overline{1}}} \\
& {\left[d_{2}, \delta H^{(1)}\right]=-\left(\mathrm{V}_{2 \overline{2}, 34}^{\mathrm{hh}, \mathrm{eh}}-\mathrm{V}_{4 \overline{2}, 32}^{\mathrm{hh}, \mathrm{eh}}\right) c_{3}^{\dagger} d_{4}^{\dagger} d_{\overline{2}}-\mathrm{V}_{\overline{1} 2, \overline{3} 3}^{\mathrm{eh}, \mathrm{ee}} c_{\overline{1}}^{\dagger} c_{\overline{3}}^{\dagger} c_{3}+\mathrm{V}_{2 \overline{2}, 43}^{\mathrm{hh}, \mathrm{he}} d_{4} c_{3} d_{\overline{2}}} \\
& {\left[c_{1}, \delta H^{(2)}\right]=\mathrm{V}_{1 \overline{2}, 34}^{\mathrm{eh}, \mathrm{eh}} c_{3}^{\dagger} d_{4}^{\dagger} d_{\overline{2}}^{\dagger}} \\
& {\left[d_{2}, \delta H^{(2)}\right]=-\mathrm{V}_{\overline{1} 2,34}^{\mathrm{e}, \mathrm{eh}} c_{3}^{\dagger} d_{4}^{\dagger} c_{\overline{1}}^{\dagger}}
\end{aligned}
$$

Finally, the Coulomb matrix elements in Eq. (A2) are defined as:

$$
\mathrm{V}_{\overline{1} 1, \overline{2} 2}^{\bar{\mu} \mu, \bar{\nu} \nu}=\int d\left(\boldsymbol{r} \boldsymbol{r}^{\prime}\right) \frac{1}{\left|\boldsymbol{r}-\boldsymbol{r}^{\prime}\right|} \Phi_{\overline{1} 1}^{\bar{\mu} \mu}(\boldsymbol{r}) \Phi_{\overline{2} 2}^{\bar{\nu} \nu}\left(\boldsymbol{r}^{\prime}\right),
$$

with

$$
\begin{aligned}
\Phi_{\overline{11}}^{\mathrm{ee}}(\boldsymbol{r}) & =\left[\phi_{\overline{1}}^{\mathrm{e}}(\boldsymbol{r})\right]^{*} \phi_{1}^{\mathrm{e}}(\boldsymbol{r}) \\
\Phi_{\overline{1} 1}^{\mathrm{hh}}(\boldsymbol{r}) & =\left[\phi_{\overline{1}}^{\mathrm{h}}(\boldsymbol{r})\right]^{*} \phi_{1}^{\mathrm{h}}(\boldsymbol{r}) \\
\Phi_{12}^{\mathrm{eh}}(\boldsymbol{r}) & =\left[\phi_{1}^{\mathrm{e}}(\boldsymbol{r}) \phi_{2}^{\mathrm{h}}(\boldsymbol{r})\right]^{*} \\
\Phi_{21}^{\mathrm{he}}(\boldsymbol{r}) & =\phi_{2}^{\mathrm{h}}(\boldsymbol{r}) \phi_{1}^{\mathrm{e}}(\boldsymbol{r}) .
\end{aligned}
$$

sponse we consider a monofrequent laser excitation of the form $\mathcal{E}_{o} e^{-i \omega t}$. Since $Y, B$, and $N$ are driven by the light field, their time dependence is also $e^{-i \omega t}$. Hence, keeping only self-interaction like processes and direct Coulomb terms, we find for $B$ and $N$ the Dyson-like equations:

\section{APPENDIX B}

$$
\begin{aligned}
& B_{12,34}=\frac{\mathrm{V}_{34, \overline{4} \overline{3}}^{\mathrm{eh}, \mathrm{he}} B_{12, \overline{3} \overline{4}}+\mathrm{V}_{34, \overline{3} \overline{4}}^{\mathrm{eh}, \overline{\mathrm{eh}}} N_{12, \overline{3} \overline{4}}}{\omega_{12}-\epsilon_{3}^{\mathrm{e}}-\epsilon_{4}^{\mathrm{h}}+i 0^{+}}+B_{12,34}^{0} \\
& N_{12,34}=-\frac{\mathrm{V}_{43, \overline{4} \overline{4} \overline{3}}^{\mathrm{he}} B_{12, \overline{3} \overline{4}}+\mathrm{V}_{43, \overline{3} \overline{4}}^{\mathrm{he}, \mathrm{eh}} N_{12, \overline{3} \overline{4}}}{\omega_{12}+\epsilon_{3}^{\mathrm{e}}+\epsilon_{4}^{\mathrm{h}}+i 0^{+}},
\end{aligned}
$$

In this Appendix we sketch the derivation of our final expression (12). Without loss of generality, in linear re-

with $\omega_{12}=\omega-\epsilon_{1}^{\mathrm{e}}-\epsilon_{2}^{\mathrm{h}}$ and

$$
B_{12,34}^{0}=\frac{\mathrm{V}_{1 \overline{1}, 34}^{\mathrm{ee}, \mathrm{eh}} Y_{\overline{1} 2}-\mathrm{V}_{2 \overline{2}, 34}^{\mathrm{hh}, \mathrm{eh}} Y_{1 \overline{2}}}{\omega_{12}-\epsilon_{3}^{\mathrm{e}}-\epsilon_{4}^{\mathrm{h}}+i 0^{+}} .
$$

Here, the infinitesimal imaginary part $i 0^{+}$has been introduced to ensure causality. Next, it turns out to be convenient to introduce the mixed representations $B_{12}(\boldsymbol{r})=\sum_{34} \Phi_{34}^{\mathrm{eh}}(\boldsymbol{r}) B_{12,34}$ and $N_{12}(\boldsymbol{r})=\sum_{34} \Phi_{43}^{\mathrm{he}}(\boldsymbol{r}) N_{12,34}$. The coupling between $B, N$ and $Y$ [third and fourth line in Eq. (9)] can then be cast to the form:

$$
\int d\left(\boldsymbol{r} \boldsymbol{r}^{\prime}\right) \frac{1}{\left|\boldsymbol{r}-\boldsymbol{r}^{\prime}\right|}\left(\Phi_{1 \overline{1}}^{\mathrm{ee}}(\boldsymbol{r})\left[B_{\overline{1} 2}\left(\boldsymbol{r}^{\prime}\right)+N_{\overline{1} 2}\left(\boldsymbol{r}^{\prime}\right)\right]-\Phi_{2 \overline{2}}^{\mathrm{hh}}(\boldsymbol{r})\left[B_{1 \overline{2}}\left(\boldsymbol{r}^{\prime}\right)+N_{1 \overline{2}}\left(\boldsymbol{r}^{\prime}\right)\right]\right)
$$

(note our neglect of exchange interactions). The sum of $B$ and $N$, which appears on the right-hand side of Eq. (B3), is obtained by summing Eqs. (B1) and transforming to the mixed representation for $B$ and $N$. We obtain:

$$
B_{12}(\boldsymbol{r})+N_{12}(\boldsymbol{r})=\int d\left(\boldsymbol{s s}^{\prime}\right) P\left(\boldsymbol{r}, \boldsymbol{s} ; \omega_{12}\right) \frac{1}{\left|\boldsymbol{s}-\boldsymbol{s}^{\prime}\right|}\left[B_{12}\left(\boldsymbol{s}^{\prime}\right)+N_{12}\left(\boldsymbol{s}^{\prime}\right)\right]+B_{12}^{0}(\boldsymbol{r}),
$$

with the polarization in random-phase approximation:

$$
P\left(\boldsymbol{r}, \boldsymbol{r}^{\prime} ; \omega\right)=\sum_{34}\left[\frac{\Phi_{34}^{\mathrm{eh}}(\boldsymbol{r}) \Phi_{43}^{\mathrm{he}}\left(\boldsymbol{r}^{\prime}\right)}{\omega-\epsilon_{3}^{\mathrm{e}}-\epsilon_{4}^{\mathrm{h}}+i 0^{+}}-\frac{\Phi_{43}^{\mathrm{he}}(\boldsymbol{r}) \Phi_{34}^{\mathrm{eh}}\left(\boldsymbol{r}^{\prime}\right)}{\omega+\epsilon_{3}^{\mathrm{e}}+\epsilon_{4}^{\mathrm{h}}+i 0^{+}}\right] .
$$


One readily recognizes that Eq. (B4) closely resembles Dyson's equation for the screened Coulomb potential W, which, in shorthand notation, reads $\mathrm{W}=\mathrm{V}+\mathrm{WPV}$. Together with:

$$
\mathrm{W}\left(\boldsymbol{r}, \boldsymbol{r}^{\prime} ; \omega\right)=\int d \boldsymbol{s} \frac{1}{|\boldsymbol{r}-\boldsymbol{s}|} K\left(\boldsymbol{s}, \boldsymbol{r}^{\prime} ; \omega\right),
$$

where $K$ is the inverse dielectric function, 13 we immediately obtain $(B+N)=K B^{0}$. Inserting this expression into Eq. (B3) we find after some straightforward calculations:

$$
\begin{aligned}
i \dot{Y}_{12} \cong & \left(\epsilon_{1}^{\mathrm{e}}+\epsilon_{2}^{\mathrm{h}}\right) Y_{12}-\frac{1}{2} \mathcal{E}_{o} e^{-i \omega t} \mathrm{M}_{12}^{\mathrm{eh}}-\left(\mathrm{V}_{1 \overline{1}, 2 \overline{2}}^{\mathrm{ee}, \mathrm{hh}}-\mathrm{V}_{12, \overline{2} \overline{1}}^{\mathrm{eh}, \mathrm{he}}\right) Y_{\overline{1} \overline{2}} \\
& +\mathrm{W}_{1 \overline{1}, 34}^{\mathrm{ee}, \mathrm{eh}}\left(\omega_{\overline{1} 2}\right) \frac{1}{\omega_{\overline{1} 2}-\epsilon_{3}^{\mathrm{e}}-\epsilon_{4}^{\mathrm{h}}+i 0^{+}} \mathrm{V}_{34, \overline{1} \overline{1}}^{\mathrm{eh}, \mathrm{ee}} Y_{\overline{1} 2}+\mathrm{W}_{2 \overline{2}, 34}^{\mathrm{h}, \mathrm{eh}}\left(\omega_{1 \overline{2}}\right) \frac{1}{\omega_{1 \overline{2}}-\epsilon_{3}^{\mathrm{e}}-\epsilon_{4}^{\mathrm{h}}+i 0^{+}} \mathrm{V}_{34, \overline{2} \tilde{2}}^{\mathrm{eh}, \mathrm{hh}} Y_{1 \tilde{2}} \\
& -\mathrm{W}_{1 \overline{1}, 34}^{\mathrm{ee}, \mathrm{eh}}\left(\omega_{\overline{1} 2}\right) \frac{1}{\omega_{\overline{1} 2}-\epsilon_{3}^{\mathrm{e}}-\epsilon_{4}^{\mathrm{h}}+i 0^{+}} \mathrm{V}_{34,2 \overline{2}}^{\mathrm{eh}, \mathrm{hh}} Y_{\overline{1} \overline{2}}-\mathrm{W}_{2 \overline{2}, 34}^{\mathrm{hh}, \mathrm{eh}}\left(\omega_{1 \overline{2}}\right) \frac{1}{\omega_{1 \overline{2}}-\epsilon_{3}^{\mathrm{e}}-\epsilon_{4}^{\mathrm{h}}+i 0^{+}} \mathrm{V}_{34,1 \overline{1}}^{\mathrm{eh}, \mathrm{ee}} Y_{\overline{1} \overline{2}},
\end{aligned}
$$

with the matrix elements of $\mathrm{W}$ defined in analogy to Eq. (A4). Finally in case of time-reversal symmetry the wavefunctions can be chosen real. Hence, the polarization $P$ is of the form: 3

$$
P\left(\boldsymbol{r}, \boldsymbol{r}^{\prime} ; \omega\right)=\int_{0}^{\infty} d \omega^{\prime} D_{o}\left(\boldsymbol{r}, \boldsymbol{r}^{\prime} ; \omega^{\prime}\right)\left[\frac{1}{\omega-\omega^{\prime}+i 0^{+}}-\frac{1}{\omega+\omega^{\prime}+i 0^{+}}\right],
$$

with $D_{o}\left(\boldsymbol{r}, \boldsymbol{r}^{\prime} ; \omega\right)=\sum_{34} \Phi_{34}^{\mathrm{eh}}(\boldsymbol{r}) \Phi_{43}^{\mathrm{he}}\left(\boldsymbol{r}^{\prime}\right) \delta\left(\omega-\epsilon_{3}^{\mathrm{e}}-\epsilon_{4}^{\mathrm{h}}\right)$, and we arrive after some straightforward algebra at Eq. (12).

* Electronic address: ulrich.hohenester@uni-graz.at

1 R. M. Dreizler and E. U. Gross, Density Functional Theory (Springer, Berlin, 1990).

2 L. Hedin and S. Lundqvist, Solid State Physics 23, 1 (1969).

3 F. Aryasetiawan and O. Gunnarson, Rep. Prog. Phys. 61, 237 (1998).

4 M. Rohlfing and S. G. Louie, Phys. Rev. B 62, 4927 (2000).

${ }^{5}$ H. Haug and S. W. Koch, Quantum theory of the optical and electronic properties of semiconductors (World Scientific, Singapore, 1993).

6 V. M. Axt and S. Mukamel, Rev. Mod. Phys. 70, 145 (1998).

7 M. Stadele, J. A. Majewski, P. Vogl, and A. Gorling, Phys. Rev. Lett. 79, 2098 (1997).

8 E. Fick and G. Sauermann, The Quantum Statistics of Dy- namic Processes (Springer, Berlin, 1990).

${ }^{9}$ F. Rossi, Semicond. Sci. Technol. 13, 147 (1998).

10 V. M. Axt and A. Stahl, Z. Physik B 93, 195 (1995).

11 In the spirit of the rotating-wave approximations, we neglect in the dynamics of $Y$ counter-clockwise rotating contributions proportional to $Y^{*}$.

12 J. W. Negele and H. Orland, Quantum Many-Particle Systems (Addison-Wesley, Redwood, 1998).

13 U. Hohenester and W. Pötz, Phys. Rev. B 56, 13177 (1997).

14 F. Bechstedt, K. Tenelsen, B. Adolph, and R. Del Sole, Phys. Rev. Lett. 78, 1528 (1997).

15 M. Bonitz, Quantum Kinetic Theory (Teubner, Stuttgart, 1998). 